\title{
Blocking Effects of CSF Spleen Vaccine on Vertical Transmission of CSFV and PRRSV Co-infection in Sows
}

\author{
Xin-Ping Liu ${ }^{1 *}$, Jiu-Xiang Yuan ${ }^{2}$, Rong-Qing Hu ${ }^{3}$, Jian-Huang $\mathrm{Zou}^{3}$, Feng-Mei Yang ${ }^{1}$, Li-Hua Yao ${ }^{1}$ \\ ${ }^{1}$ School of Life Science, Jiangxi Science \&Technology Normal University, Nanchang, 330013, China \\ ${ }^{2}$ Livestock and Poultry Experiment Center of Nanchang City, Nanchang, 330009, China; \\ ${ }^{3}$ Animal Husbandry Bureau of Jinxian County, Nanchang, 331700, China \\ "Corresponding author: Xin-Ping Liu, E-mail: nckdliuxp@sina.com
}

\begin{abstract}
To investigate the blocking effects of classical swine fever (CSF) spleen vaccine on vertical transmission of classical swine fever virus (CSFV) and porcine reproductive and respiratory syndrome virus (PRRSV) co-infection in sows. Sows infected CSFV+PRRSV were selected from three large-scale pig farms and they were randomly divided into group I and group II. The sows in the group I were set as experimental group and vaccinated with CSF spleen vaccine at a 2 times normal dose per pig; and sows in group II were set as control group and vaccinated with CSF cell vaccine at a 6 times normal dose per pig. The antigens of piglets were detected by enzyme-linked immunosorbent assay (ELISA) method. Our results revealed that the blocking rate on the antigen of piglets in the experimental group $(79.25 \%, 42 / 53)$ was significantly higher than that in the control group $(50.94 \%, 27 / 53)$ ( $\mathrm{p}<0.01)$. Collectively, these results demonstrate that CSF spleen vaccine has a good blocking effects on vertical transmission of CSFV+PPRSV coinfection in sows.

Index Terms - classical swine fever virus (CSFV), porcine reproductive and respiratory syndrome virus (PRRSV), sows, vertical transmission
\end{abstract}

\section{Introduction}

Classical swine fever (CSF), one of the main infectious diseases in swine industry, is caused by classical swine fever virus (CSFV) [1]. Porcine reproductive and respiratory syndrome (PRRS) is also a contagious diseases in swine industry infected by porcine reproductive and respiratory syndrome virus (PRRSV) [1,2]. Recent studies have shown that the reproductive disorders could be induced by coinfection with various pathogenic viruses like CSFV and PRRSV [1]. Moreover, previous studies also revealed that this co-infection pathological phenomena was become more and more widespread with the development of the swine industry [1- 4].

In recent years, the prevalence of the CSF disease was controlled due to the mandatory vaccination policy on CSF in China. Nowadays, the breeding sows usually present CSFV persistent infection and develop as a "chronic atypical syndrome" disease, which in turn account as an important underlying pathological mechanism to the CSFV persistent infection in most large pig farms [5]. "Chronic atypical syndrome" generally refers to latent infection in pregnant sows caused by natural infection with low or intermediate CSFV [4]. The relationship between infected sows and CSFV vertical transmission in Guangdong and Fujiang Provinces has shown that the CSFV positive rate of piglets produced by infected sows was $66 \%-100 \%$ [4].

Typically, culling out the infected sows or vaccination are the two main methods to control and eradicate CSF [6]. However, it seems impossible to control the CSF via culling out all infected sows due to the more and more widespread of the infected sows in most pig farms so far. Thus, the way of vaccine prevention on the CSF becomes more and more important. For achieving the maximum vaccine protective effect on sows, choosing the way of obtaining an optimal vaccine and draw up a reasonable immune procedure is a critical course [6,7]. In the present study, the blocking effects of CSF spleen vaccine and CSF cell vaccine on vertical transmission of CSFV+PRRSV co-infection in sows were studied side by side.

\section{Materials and Methods}

A. Materials

293 serum samples were collected from sows in five large scale pig farms of Jiangxi Province from October to November in 2012. A total of 106 serum samples from piglets produced by infected sows were collected randomly from March to April in 2013.

HerdChek CSFV serum antigen detection kits was purchased from IDEXX (IDEXX, USA). rTaq DNA ( $5 \mathrm{u} / \mu \mathrm{l})$, dNTP, and DNA Maker DL 2000 were purchased from Dalian Baosheng Biotechnology Co., Ltd. (Dalian, China). Trypsin, DMEM were purchased from Gibco Co. (Gibco BRL, USA). The primers were designed and synthesized base on PRRSV genome sequence via Primer 5.0 software. The upstream primer 5'-ATGGCCAGCCAGTCAATCA-3' and the downstream primer 5'- TCGCCCTAATTGAATAGGTG-3' were synthesized by the Shanghai Biological Engineering Co., Ltd. And the primers at concentration of $20 \mathrm{pmol} / \mu \mathrm{l}$ was employed in the experiment.

\section{B. Animals grouping and immunization}

6 infected multiparous sows were selected from three large representative pig farms by HerdChek CSFV serum antigen detection kits, and they were randomly divided into group I, group II, three in each group. At $10-15 \mathrm{~d}$ before mating, the sows in the group I were vaccinated with CSF spleen vaccine at a 2 times normal dose per pig; those in the group II were vaccinated with CSF cell vaccine at a dose of 6 
times normal dose per pig. The blood samples were collected from piglets. The CSF antigens were detected by CSFV detection ELISA kit.

\section{Samples detection}

Antigen detection was achieved according to the instructions of HerdChek CSFV serum antigen detection kits. Firstly, the CSFV monoclonal antibodies were coated on the microplate, and then the CSFV antigens were captured by the specific antibodies and labeled by the horseradish peroxidase (HRP), the uncombined HRP were washed. Secondly, the substrates and chromogen solution were added, then the substrates transformed into substances which could react with the chromogen to produce blue products. After adding termination solution, the mixture showed yellow. Finally, the $A_{450}$ or $A_{450 / 650}$ of samples was detected by spectrophotometer. The corrected $A_{450}$ was calculated according to the sample $A_{450}$ and the control corrected $A_{450}$.

\section{Judging standards}

The results must meet following standards: $\mathrm{PC}-\mathrm{NC} \geqslant$ $0.015 \mathrm{~A}, \mathrm{NC} \leqslant 0.250 \mathrm{~A}$; which, $\mathrm{PC}$ strands for average absorbance of positive control, NC strands for average absorbance of negative control. When corrected $A_{450} \geqslant 0.300$, the tested antigen was positive.

Average absorbance of positive control (PC):

$$
\mathrm{PC} \bar{X}=\left(\mathrm{PC} 1 A_{450}+\mathrm{PC} 2 A_{450}\right) / 2
$$

Average absorbance of negative control (NC):

$$
\mathrm{NC} \bar{X}=\left(\mathrm{NC} 1 A_{450}+\mathrm{NC} 2 A_{450}\right) / 2
$$

Corrected $A_{450}$ :

$$
\mathrm{S}-\mathrm{N}=\text { sample } A_{450} \text {-NC } \bar{X} A_{450}
$$

\section{Results}

A. CSF antigen positive rates from five pig farms' sows

The positive rate of CSF antigens in 293 serum samples collected from sows in five large scale pig farms was $15.36 \%$ $(45 / 293)$, and the positive rate in each pig farm was $18.18 \%$ (12/66), $16.13 \%$ (10/62), $15.80 \%$ (9/57), $14.55 \%$ (8/55) and $11.32 \%$ (6/53), respectively (Table.1), indicating that the coinfection of sows with CSFV+PRRSV were widespread not only in the above 5 pig farms.

TABLE I CSFV antigen positive rates of sows from five pig farms

\begin{tabular}{|c|c|c|c|}
\hline Farms & Serum & Positive serum & Positive rate (\%) \\
\hline Farm 1 & 66 & 12 & 18.18 \\
\hline Farm 2 & 62 & 10 & 16.13 \\
\hline Farm 3 & 57 & 9 & 18.80 \\
\hline Farm 4 & 55 & 8 & 14.55 \\
\hline Farm 5 & 53 & 6 & 11.32 \\
\hline Total & 293 & 45 & 15.36 \\
\hline
\end{tabular}

\section{B. Detecting result of CSF antigen on piglets from three pig farms}

The detecting results of CSF antigens in 106 sera from piglets in three pig farms were shown in Table 2. The chisquare test revealed that the antigen blocking rate on piglets in the experimental group $(79.25 \%, 42 / 53)$ was significantly higher than that in the control group $(50.94 \%, 27 / 53)$ $(\mathrm{p}<0.01)$, suggesting that CSF spleen vaccine has a good blocking effects on vertical transmission of CSFV+PPRSV coinfection in sows.

TABLE II Detecting result of CSF antigen on piglets from three pig farms

\begin{tabular}{|c|c|c|c|c|c|c|}
\hline \multirow{2}{*}{ Farms } & \multicolumn{3}{|c|}{ Experimental group } & \multicolumn{3}{c|}{ Control group } \\
\cline { 2 - 7 } & Serum & $\begin{array}{c}\text { Negative } \\
\text { serum }\end{array}$ & $\begin{array}{c}\text { Blocking } \\
\text { rate (\%) }\end{array}$ & Serum & $\begin{array}{c}\text { Negative } \\
\text { serum }\end{array}$ & $\begin{array}{c}\text { Blocking } \\
\text { rate (\%) }\end{array}$ \\
\hline Farm 1 & 17 & 13 & 76.47 & 17 & 8 & 47.06 \\
\hline Farm 2 & 19 & 15 & 78.95 & 19 & 10 & 52.63 \\
\hline Farm 3 & 17 & 14 & 82.35 & 17 & 9 & 52.94 \\
\hline Total & 53 & 42 & 79.25 & 53 & 27 & 50.94 \\
\hline
\end{tabular}

\section{Discussion}

"Chronic atypical syndrome" caused by CSFV is one of the major reasons for CSF prevalence, and it is also the most easily overlooked disease type in CSF [4].The CSF persistent infection is rooted in the infected sows without any clinical symptoms. The virus can vertically infect the fatal pig though placenta. If the piglets are hold as prepubertal gilts, they will become infected hidden killers because of immune tolerance, which leads to a vicious circle. PRRS, as one of the most serious immunosuppressive diseases induced by PRRSV, is characterized by a delayed and defective adaptive hog immune response $[8,9]$. Previous studies have shown that the first outbreak and prevalence of PRRS happened in China has caused heavy economic losses in many pig-producing regions [10-11]. Moreover, studies also revealed that a co-infection pathological disease caused by CSFV+PRRSV was become more and more prevalence in many pig-producing regions, which usually appears "chronic atypical syndrome" and reproductive disorders [1-4].

Therefore, choosing the way of obtaining an optimal vaccine and draw up a reasonable immune procedure is a critical course [6-7]. CSF cell vaccine, is produced by using CSFV inoculated into calves' testicular cells, which only possesses specific immune function [4]. CSF spleen vaccine, is typically produced through following procedures: Chinese hog cholera lapinised virus (HCLV) $\mathrm{C}$ strains were inoculated into healthy adult rabbits, then the spleens and mesenteric lymph nodes were harvested from the infected rabbits and prepared to emulsion. The emulsion was dried by freezing in a high vacuum to prepare the CSF spleen vaccine. The virus in 450fold diluted CSF spleen vaccine can cause the immune response in rabbits. The immunogenicity of the CSF spleen vaccine is 5 times higher than cell vaccine and the antigen content is 4 times higher than international standard [4]. The 
CSF spleen vaccine contains lymphoid tissue active factors which have the non-specific immune enhancing activity.

In the present, in order to obtain an optimal vaccine and draw up a reasonable immune procedure, the blocking effects of CSF spleen vaccine and CSF cell vaccine on vertical transmission of CSFV+PRRSV co-infection in sows were studied side by side. Our results revealed that the blocking rate on the antigen of piglets in the experimental group (vaccinated with CSF spleen vaccine) $(79.25 \%, 42 / 53)$ was significantly higher than that in the control group (vaccinated with CSF cell vaccine) $(50.94 \%, 27 / 53)(\mathrm{p}<0.01)$. Collectively, these results demonstrate that CSF spleen vaccine has a good blocking effects on vertical transmission of CSFV+PRRSV co-infection in sows.

\section{Acknowledgment}

This work was supported by Science and Technology Projects of Jiangxi science and technology Department (20122BBF60068)

\section{References}

[1] Z.-Y. Zhuge, Y.-H. Zhu, P.-Q. Liu, X.-D. Yan, Y. Yue, X.-G. Weng, R. Zhang, J.-F Wang, "Effects of astragalus polysaccharide on immune responses of porcine PBMC stimulated with PRRSV or CSFV," PloS One, vol. 7, no. 1, pp. e29320, 2012.

[2] Y. Zhou, J. Bai, Y. Li, X. Wang, X. Wang, P. Jiang, "Suppression of immune responses in pigs by nonstructural protein 1 of porcine reproductive and respiratory syndrome virus," Can J Vet Res, vol. 76, no. 4, pp. 255-260, 2012.
[3] Q.-L. Truong, T.-W. Seo, B.-I. Yoon, H.-C. Kim, J.-H. Han, T.-W. Hahn, "Prevalence of swine viral and bacterial pathogens in rodents and stray cats captured around pig farms in Korea," $J$ Vet Med Sci, in press.

[4] X. Liu, L. Gao, J. Xu, X. Feng, J. Huang, F. Yang, "Blocking effects of spleen vaccine on vertical transmission of classical swine fever virus in sows," Animal Husbandry and Feed Science, vol. 2, no. 5, pp. 24-25 2010.

[5] X. Lu, J. Liu, W. Fu, J. Zhou, Y. Luo, X. Ding, Y. Liu, Q. Zhang, "Genome-wide association study for cytokines and immunoglobulin $\mathrm{G}$ in swine," PloS One, vol. 8, no. 10, pp. e74846, 2013.

[6] H.-J. Qiu, G.-Z. Tong, R.-X. Shen, "The lapinized Chinese strain of classical swine fever virus: a retrospective review spanning half a century," Scientia Agricultura Sinica, vol. 38, no. 8, pp. 1675-1685, 2005. (in Chinese)

[7] G. Franzoni, N.-V. Kurkure, D.-S. Edgar, H.-E. Everett, W. Gerner, K.-B. Bodman-Smith, H.-R. Crooke, S.-P. Graham, "Assessment of the phenotype and functionality fo porcine CD8 $\mathrm{T}$ cell responses following vaccination with live attenuated classical swine fever virus (CSFV) and virulent CSFV challenge," Clin Vaccine Immunol, vol. 20, no. 10, pp. 1604-1616, 2013.

[8] L.-C. Miller, W.-W. Laegreid, J.-L Bono, C.-G. Chitko-Mckown, J.-M. Fox, "Interferon type I response in porcine reproductive and respiratory syndrome virus-infected MARC-145 cells," Arch Virol, vol. 149, no. 12, pp. 2453-2463, 2004.

[9] L.-C. Miller, K.-M. Lager, M.-E. Kehrli, "Role of toll-like receptors in activation of porcine alveolar macrophages by porcine reproductive and respiratory syndrome virus," Clin Vaccine Immunol, vol. 16, no. 3, pp. 360-365, 2009.

[10] Y. Li, X. Wang, K. Bo, X. Wang, B. Tang, B. Yang, W. Jiang, P. Jiang, "Emergence of a highly pathogenic porcine reproductive and respiratory syndrome virus in the mid-eastern region of China," Vet $J$, vol. 174, no. 3, pp. 577-584, 2007.

[11] K. Tian, X. Yu, T. Zhao, Y. Feng, Z. Cao, C. Wang, et al., "Emergence of fatal PRRSV variants: unparalleled outbreaks of atypical PRRS in China and molecular dissection of the unique hallmark," PloS One, vol. 2, no. 6, pp. e526. 